\title{
A REVIEW ON ROLE OF TRPV CATION CHANNELS
}

\author{
${ }^{1}$ Deep Sharma, ${ }^{2}$ Rekha Rana, ${ }^{3}$ Kiran Thakur
}

Department of Pharmaceutical Sciences

1,2 Associate Professor, ${ }^{3}$ Assitant Professor DDM College of Pharmacy, Una, Himachal Pradesh, India, Pincode-177213

Article Info: Received 5 February. 2021; Accepted 5 March 2021

DOI: https://doi.org/10.32553/jbpr.v10i2.857

Corresponding author: Kiran Thakur

Conflict of interest statement: No conflict of interest

\begin{abstract}
The mammalian branch of the Transient Receptor Potential (TRP) superfamily of cation channels consists of 28 members. They can be subdivided in six main subfamilies: the TRPC ('Canonical'), TRPV ('Vanilloid'), TRPM ('Melastatin'), TRPP ('Polycystin'), TRPML ('Mucolipin') and the TRPA ('Ankyrin') group. The TRPV subfamily comprises channels that are critically involved in nociception and thermo-sensing (TRPV1, TRPV2, TRPV3, TRPV4) as well as highly $\mathrm{Ca}^{2+}$ selective channels involved in $\mathrm{Ca}^{2+}$ absorption/ reabsorption in mammals (TRPV5, TRPV6). In this review we summarize fundamental physiological properties of all TRPV members in the light of various cellular functions of these channels and their significance in the various diseases.
\end{abstract}

Keywords: TRP channels-Vannilloid-TRPV-nociception-thermosensing-Ca ${ }^{2+}$ homeostasissystemic diseases.

\section{Introduction}

The Transient receptor potential vanilloid (TRPV) family of ligand gated ion channels represents a group of non-selective cation channels. These are reported for their multiformity of function in pain, thermo-, mechano-, and osmosensation and also play key roles in calcium homeostasis (Senning and Gordon 2011). TRPV channels are found across the plasma membrane of human, various animal cell types and some fungi. Some of these channels are also found in organellar membranes and also having function in cellular as well as systemic osmotic homeostasis in vertebrates (Liedtke 2006). Many TRP channels having polymodality signal integrators which activate cellular signaling pathways through non-selective cation flux (e.g. $\mathrm{Ca}^{2+}$, $\mathrm{Mg}^{2+}, \quad$ and $\quad \mathrm{Na}^{+}$). Post-translational modifications can leads to the activation of TRPV channel such as phosphorylation or binding of regulatory molecules to intracellular domains (Ramsey, Delling et al. 2006). In TRP channel family, from the Caenorhabditiselegans, first identified TRP channel of the TRPV family was OSM-9 (osmotic avoidance abnormal family member 9). OSM-9 is required for olfaction, mechanosensation and olfactory adaptation. The other TRPV channels in C.elegans are OCR-1 to OCR-4 (osm-9/capsaicin receptor related), for a total of five.

In the dorsal root ganglion (DRG), several TRP channels located respond to nociceptive stimuli to cause pain. TRPV channels have been incriminate in several physiological and pathological processes such as cancer, genetic disorders, and other uncommon diseases, and 
not only limited to somatosensation. The transient receptor potential (TRP) channels are tetramericcation channels superfamily of sensory channels widely expressed across every tissue and cells type. These TRP channels show different permeability and selectivity to monovalent and divalent cations, whereas most trivalent cations act as channel blockers. Physiologically, cation permeability selectivity is important. Since cations play important roles in cell regulation and function, such as fertilization, muscle contraction, or exocytosis. In this review, we have discussed the physiological as well as pathological role of TRPV channels and implications in various diseases. Here, the Transient Receptor Potential Vanilloid (TRPV) channel represent themselves a subject for discussion, one of the most curious and fascinate members of TRP superfamily, with a slenderness of data concerning the endogenous function of these channel.

\section{Classification}

TRP channels were originally spot within the rhabdomeres of the photoreceptor in Drosophila (Minke 1977) and the first member was cloned in the late 80s (Montell and Rubin 1989). After the potassium channel family, it is the second largest family of voltage-gated-like ion channels (Almén, Nordström et al. 2009).

Drosophila TRP channels discovered numerous homologous proteins, both in invertebrate species such as nematodes, and in vertebrate species (Venkatachalam and Montell 2007). On the basis of their structure, function and amino acid sequence identity and similarity, members of the TRP channel family divided into seven subfamilies: TRPA (ankyrin), having 1 human member; TRPC (canonical), having 6 human members, plus 1 human pseudogene; TRPM (melastatin) having 8 human members; TRPML (mucolipin) having 3 human members; TRPN (NompC) having no human members, TRPP (polycystin) having 3 human members, and TRPV (vanilloid) having 6 human members. There is one more distantly related family, TRPY, found in yeast. The TRPC, TRPM, TRPV, TRPA, and TRPN subfamilies, called as Group I TRP channels, resemble one another more closely than TRPP or TRPML subfamilies, which are classified as Group II. Almost all the TRP channels are selective for cations, whereas their degree of judgment among cations can vary (see Table 1.)

TRPV proteins function as tetrameric and each protomer contains six transmembrane segments (S1 to S6), with a pore-forming loop between S5 and S6. These are flanked by two cytoplasmic domains; a large $\mathrm{N}$-terminal domain containing ankyrin repeats and a shorter C-terminal domain. The larger $\mathrm{N}$ terminal ankyrin repeat domain (ARD) binds to small molecules and proteins (Inada and Gaudet 2011). The TRPV subfamily is divided in two groups based on amino acid sequence homology, TRPV1-4 and TRPV5-6. The group formed by TRPV1-4 share low sequence identity $(\sim 20 \%)$, but high identity with the TRPV1-4 group ( 75\%) (Montell 2005; Clapham 2007). TRPV5 and TRPV6 are highly selective for calcium channels important for general $\mathrm{Ca}^{2+}$ homeostasis and heterotetramers between them (Hellwig, Albrecht et al. 2005)(see Table. 2). All four channels within the TRPV1-4 group show sensory signalling, polymodality, temperature-invoked currents when manifest in heterologous cell systems, ranging from activation at $\sim 25^{\circ} \mathrm{C}$ for TRPV4 to $\sim 52^{\circ} \mathrm{C}$ for TRPV2 but non-selective cation channels. TRPV1-4 channels heteromerization has been documented, mostly in heterologous expression systems. Some channels such as TRPV5 and TRPV6 are highly selective for $\mathrm{Ca}^{2+}$ (Owsianik, Talavera et al. 2006)(see Table. 2)

\section{Expression and Location}

As a family, TRP channels are broadly expressed in mammalian tissues. In fact, at least one family member is likely expressed in every cell of the body and often more. Moreover, these channels can also be stimulated by a number of heterogeneous stimuli, including endogenous as well as exogenous chemical ligands, physical stimuli such as mechanical force and high temperature, free cytosolic $\mathrm{Ca}^{2+}$ ions, depletion of 
endoplasmic reticulum $\mathrm{Ca}^{2+}$ stores, and many others. These channels have been linked to numerous physiological functions (see fig 2).

\section{Expression of TRPV channel in brain}

Within the central nervous system, all the TRPV channel subfamilies expressed in humans have been implicated in important functions (Verkhratsky, Reyes et al. 2013). Examples include TRPV1, TRPV2 function for the neurotransmitter release, TRPV1 and TRPV4 plays role in osmoregulation (see fig 2).

\section{Expression of TRPV channel in cardiac}

TRPV2 and TRPV4 are expressed within the heart and blood vessels of endothelial or muscle cells, where they regulate vascular tone and permeability, as well as cardiac contractility (Inoue, Morita et al. 2004) (see fig 2).

\section{Expression of TRPV channel in kidney}

Within the kidneys and lower urinary tract, TRPV channels contributes to the number of processes (Everaerts, Gevaert et al. 2008; Birder and Andersson 2013). TRPV4 regulates the Nephron osmoregulatory function. Calcium and magnesium uptake is regulated by TRPV5 and TRPV6. Within the urinary bladder epithelium and bladder afferents, detection of stretch and irritation is regulated by the TRPV1 and TRPV4 (see fig 2).

\section{Expression of TRPV channel in GIT}

In the gastrointestinal tract, many TRPV channels are expressed (Holzer 2011). For example, calcium absorption in the gut is stimulated by the TRPV6, which is a key effector of vitamin D. Although, TRPV2 appears to regulate gastrointestinal motility and is expressed in enteric neurons. There is generous stimulation of the gastrointestinal tract by external sensory afferents that articulate TRPV1 channels. Mucosal blood flow and sensitivity to luminal distension such processes are controlled by these neuronal channels. Inside the exocrine portion of the gastrointestinal tract, TRPV4 regulate saliva production and/or pancreatic acinar cell secretion, when the modulation of intracellular calcium levels and the regulation of aquaporin water channels occurred (see fig 2).

\section{Expression of TRPV channels in immune system}

Within the immune cell processes confirmed the involvement of TRPV channels. TRPV2 leads to the cytokine release, lysosomal acidification, and microglial phagocytosis (Santoni, Farfariello et al. 2013). TRPV channels are also contributed to function in skin keratinocytes and primary sensory afferents. E.g. from the keratinocytes, the release of immune modulatory molecules such as TSLP and endothelin-1 mediated by the TRPV4 (Moorea, Cevikbasb et al. 2013; Wilson, Thé et al. 2013), whereas the neurogenic release of neuropeptides from sensory afferents mediated through TRPV1, which can leads to regulate recruitment of immune cells and promote tissue swelling (Geppetti, Nassini et al. 2008; Bautista, Pellegrino et al. 2013)(see fig 2).

\section{Expression of TRPV channels in reproduction}

In the reproduction and embryonic development, TRPV channels play an important role. Differentiation of the skin and hairs are controlled by the TRPV3 channels, and keratinocyte production of EGF receptor ligands are also regulated by TRPV3 channels (Cheng, Jin et al. 2010), whereas In human adipocytes differentiation, the TRPV channels involved are TRPV2 and TRPV4 (Che, Yue et al. 2014) (see fig 2).

\section{TRPV-1}

TRPV1, nonselectivecation channel with high permeability to $\mathrm{Ca}^{2+}$ ions, also known as the capsaicin receptor. It is the first cloned member of the TRPV family (Caterina, Leffler et al. 2000). TRPV1 consist of six transmembrane domain and pore region located between the fifth and sixth transmembrane segments. As a nociceptor, activity of TRPV1 can be regulated by a diverse range of physical and chemical stimuli, including the binding of nucleotides, local concentration of phospholipid, capsaicin, heat (approximately $43^{\circ} \mathrm{C}$ ), protons, 
bradykinin, nerve-growth factor, anandamide, prostaglandins, adenosine and ATP, polyamines and venoms from fish and insects (Gharat and Szallasi 2007). TRPV1 became a popular target for pain control for several reasons. First, the agents or physical stimuli that activate TRPV1 are those cause pain sensation in humans or rodents (Knotkova, Pappagallo et al. 2008). TRPV1 activity is indeed up-regulated in inflammatory conditions. Conversely, compounds that desensitize or antagonize TRPV1 alleviate pain-like behaviors or pain in rodents and human, respectively (Wong and Gavva 2009).

\section{Activation}

TRPV1 is activated by temperature greater than $43^{\circ} \mathrm{C}\left(109^{\circ} \mathrm{F}\right)$, anandamide, acidic conditions, allylishothiocyanate, capsaicin, low $\mathrm{pH}$, resiniferatoxin, heat, and eicosanoids. In knockout mice, TRPV1 has been shown to play an important role in thermosensation(FerrerMontiel, Fernández-Carvajal et al. 2012).

\section{Role of TRPV1 in GIT}

TRPV1 expression has also been found in the parietal cells. In the distal colon and rectum, number of TRPV1-immunoreactive axons have been found, whereas in the transverse and proximal colon, they are barely expressed (Matsumoto, Kurosawa et al. 2009). In the rat gastric epithelial cell lines and rat gastric wall (RGM-1), expression of TRPV1 mRNA has been detected in the cells (Geppetti and Trevisani 2004).

TRPV1 can be used as a potential target for the treatment of gastroesophageal reflux disease (GERD) as the TRPV1 present on the afferents and innervating the human esophagus mucosa. Also its expression increased in disease conditions (Matthews, Aziz et al. 2004; Bhat and Bielefeldt 2006). After exposure to the acid, TRPV1 knockout mice show a slighter degree of esophagitis as compared to wild-type controls (Fujino, de la Fuente et al. 2006). In response to noxious rectal distension and inflammation, visceral pain is mediated through the TRPV1-positive neurons (Jones, $\mathrm{Xu}$ et al. 2005; Spencer, Kerrin et al. 2008; Wang, Wang et al. 2008). It has been reported that inhibition of TRPV1 by using the antagonists and silencing by RNA interference leads to ameliorate visceral pain in rats (Jones, $\mathrm{Xu}$ et al. 2005; Christoph, Grünweller et al. 2006; Sugiura, Bielefeldt et al. 2007).

It has been observed that patients with IBD having increased level of TRPV1immunoreactivity in colonic sensory afferents (Yiangou, Facer et al. 2001). In the rat model of irritable bowel syndrome, during the neonatal period it has been found that TRPV1 antagonists leads to prevent the development of visceral hypersensitivity initiated by acetic acid treatment (Winston, Shenoy et al. 2007; Keszthelyi, Troost et al. 2013).

\section{Role of TRPV1 in urinary system}

In the urinary system, TRPV1 having expression in neuronal and nonneuronal cells. These includes bladder, ureter, renal pelvis, and urethra (Bishnoi, Bosgraaf et al. 2011; Avelino, Charrua et al. 2013). In rat and human urothelial cells, agonists and physiological stimuli (heat and low $\mathrm{pH}$ ) leads to the activation of TRPV1 and cause ATP release and calcium influx (Avelino, Charrua et al. 2013). Intravesical administration of capsaicin or RTX in the patients with neurogenic detrusor overactivity disorder leads to increases bladder capacity and partially restores continence (Lazzeri, Beneforti et al. 1997; Silva, Silva et al. 2007). In patients with detrusor overactivity, intravesical administration of RTX has been found to be safe and effective safe (Silva, Rio et al. 2000; Silva, Avelino et al. 2001; Watanabe, Yokoyama et al. 2004; Silva, Silva et al. 2005).

\section{TRPV2}

TRPV2 is second described member of TRPV ion channel family. It is initially characterized as a noxious heat sensor. There are so many regulatory interactions between signalling molecules and TRPV2 cytoplasmic domains have been investigated. In the RBL2H3 mast cell line, by using a two-hybrid screening, the interaction between TRPV2 and recombinase 
gene activator (RGA) have been identified (Barnhill, Stokes et al. 2004).

However, TRPV2 physiological role in temperature sensing has not been demonstrated. In TRPV2 knockout mice, the thermal responses have been similar to the wild type mice (Park, Vastani et al. 2011). The role of TRPV2 in various osmo or mechanosensory mechanisms is believed to be increased. The TRPV channels mechanosensory function seems to be maintained through evolution. TRPV2 orthologs have been cloned by two labs simultaneously. Based on TRPV2 homology to TRPV1, TRPV2 channel of human and rat were cloned, and named vanilloid-receptor-like protein 1 (VRL-1) (Caterina, Rosen et al. 1999). Mouse TRPV2 was cloned and stimulation of cells by insulin-growth-factor-1 (IGF-1) induces calcium current, based on this fact named growth-factor regulated channel (GRC) (Kanzaki, Zhang et al. 1999). Osm-9like TRP channel 2 (OTRPC2) infrequently has been referred to TRPV2 (Harteneck, Plant et al. 2000). In vitro, Heterotetramerization of TRPV2 with TRPV1 has been report in HEK cells (Hellwig, Albrecht et al. 2005; Rutter, Ma et al. 2005; Cheng, Yang et al. 2007). In vivo, TRPV1 and TRPV2 shown to tightly colocalize in rat DRG (Hellwig, Albrecht et al. 2005; Liapi and Wood 2005; Rutter, Ma et al. 2005). However, TRPV2 heterotetramerization physiological roles have yet to be determined.

\section{Structure and evolution of TRPV2}

TRPV2 present in higher concentration within mammalian orthologs. Specific TRPV2 orthologs present in tetrapod vertebrates, but not found in fish, where there is a TRPV $1 / 2$ isoform present. A evolutionary hypothesis suggest that TRPV1/2, duplicated generating two genes is a common ancestor between TRPV1 and TRPV2 (Saito and Shingai 2006). With time, the gene duplication will become confusing. The sequence features of TRPV2 are best understood when compared with the other TRPV subfamily members. TRPV2 consists of a large N-terminal cytoplasmic domain ( $>390$ residues) and a $\mathrm{C}$-terminal cytoplasmic domain ( $\sim 100$ residues). The $\mathrm{N}$-terminal cytoplasmic domain having six transmembrane segments ( $\sim 250$ residues) containing a pore-forming loop. In TRPV2 channel, N-terminal cytoplasmic region further subdivided into a membrane-proximal linker region and a distal $\mathrm{N}$-terminal region, an ankyrin repeat domain. The first $\sim 60-70$ residues comprises by the distal N-terminal cytoplasmic region. In the first crystal structure obtained for a human TRPV2 N-terminal region, the distal $\mathrm{N}$ terminal cytoplasmic region were not ordered and therefore not visible (McCleverty, Koesema et al. 2006). As assessed in vitro in rat TRPV2, channel functions did not alter when there is deletion up to $65 \mathrm{~N}$-terminal residues, whereas plasma membrane expression is reduced in HEK293 when deletion of 83 or more residues occurred (Neeper, Liu et al. 2007).

The ankyrin repeat domain (ARD) of TRPV2 includes approximately $\sim 70-320$ residues, which contains six ankyrin repeats. This soluble domain has yielded the information currently available for TRPV2: the crystal structure of the TRPV2-ARD in human and rat were published nearly simultaneously (Jin, Touhey et al. 2006; McCleverty, Koesema et al. 2006). Each TRPV2 ankyrin repeat (ANK1-6) is composed of the two opposite helices (inner and outer) followed by a loop region linked to a $\beta$-hairpin structure. In the structure, these motifs are arranged linearly, with repeats packing side-by-side. The $\beta$-hairpin structures foretelling out $\sim 90$ degrees comparative the helical axes. Every ankyrin repeat is characteristically rotated a few degrees counter clockwise relative to the earlier repeat, leading to an overall helical arrangement of the repeats (Michaely, Tomchick et al. 2002). The generated concave surface is often used in protein-ligand interactions and the function of ARD-containing proteins are modulated. TRPV2 channels having a lesser grade of conservation than TRPV1, when the sequence conservation is mapped onto the molecular surface of the ARD within a set of orthologus sequences. The largely conserved region on the TRPV2-ARD surface does map to the concave surface, moderately overlapping with the larger 
conserved region on the TRPV1-ARD. TRPV2 transmembrane domain (TMD; residues $\sim 390$ and $\sim 640$ ) is predicted to contain a poreforming loop between S5 and S6 and a six transmembrane segments (S1-S6) (Krogh, Larsson et al. 2001). TRPV2 residues in the S4S5 linker region may not be significant for voltage sensitivity and no such effect has been observed when mutation of the analogous residues takes place in the TRPV2 S4-S5 linker (Boukalova, Marsakova et al. 2010). In the S5S6 loop, there is the presence of an $\mathrm{N}$ glycosylation consensus motif (NXT/S) in the TRPV2 TMD. While the first asparagine is found only in rat and hamster TRPV2. In rat F11 DRG cells, rat and mouse TRPV2 heterologously expressed in a glycosylated form in the plasma membrane whereas the endogenous TRPV2 isoform (rat) remains in the cytoplasm in a non-glycosylated form (Jahnel, Bender et al. 2003). From these results we can conclude that glycosylation having a role in TRPV2 trafficking, as has been shown same results for other TRPs (Erler, Al-Ansary et al. 2006; Morenilla-Palao, Pertusa et al. 2009). From the other reports we reported that the TRPV2 glycosylation may play an important role under the modulation of the Klotho factor (Lin and Sun 2012).

The TRPV2 selectivity filter and pore-forming domain is highly conserved which is located in the S5-S6 region. TRPV2 having different divalent/monovalent cation permeability. Due to aspartate-to-glutamate substitution in TRPV2, this may leads to the difference in selectivity. Mutagenesis studies in the poreforming region has been indicated that in HEK cells, mouse TRPV2 heterologously expressed may be constitutively active, which may leads to inducing high cytotoxicity, since a altered in the charged mutant (E594K) reduced cytotoxicity (Penna, Juvin et al. 2006). In rat TRPV2, the C-terminal cytoplasmic domain spanning residues 639 to 761 contains significant characters for the assemblage of the channel, such as calmodulin-binding domains, oligomerization (TRP domain) and phosphatidylinositol 4-5-bisphosphate (PIP2) binding (García-Sanz, Fernández-Carvajal et al.
2004; Mercado, Gordon-Shaag et al. 2010; Holakovska, Grycova et al. 2011). There are number of regulatory interactions between various signalling molecules and TRPV2 cytoplasmic domains have been investigated. In the RBL2H3 mast cell line, an interaction of TRPV2 with recombinase gene activator (RGA) (Barnhill, Stokes et al. 2004) was identified via a two hybrid screening. These two hybrid screening use the first $\sim 390$ residues of human TRPV2 which is equivalent to the whole N-terminal cytoplasmic region. Later on, it was confirmed that a physiological complex having both TRPV2 and RGA to the plasma membrane is essential for the cAMP-driven trafficking of TRPV2. It is remain unclear that whether RGA and TRPV2 interact directly (Stokes, Wakano et al. 2005).

In the Gordon Lab, initially predicted a Cterminal TRPV2 calmodulin-binding domain and later characterized in vitro (GordonShaag, Zagotta et al. 2008; Mercado, GordonShaag et al. 2010). Further characterized this calmodulin-binding domain and is restricted to the 654-683 region of human TRPV2 channels, nearly completely overlapping with the TRP domain (Holakovska, Grycova et al. 2011). Although calmodulin binding to a $\mathrm{C}$-terminal fragment of TRPV2 (residues 684-753) in a $\mathrm{Ca}^{2+}$ dependent manner, the Gordon lab could not explain that in TRPV2 desensitization, calmodulin plays a major role (Mercado, Gordon-Shaag et al. 2010). In the TRP channel interacting protein (TRIP) database, a few additionally characterized TRPV2-interacting proteins have been cataloged(Shin, Shin et al. 2010; Shin, Shin et al. 2012).

\section{Physiology and signalling pathways of TRPV2}

TRPV2 is a homotetrameric N-glycosylated protein that translocate from intracellular membrane compartments to the plasma membrane after stimulation of the phosphatidylinositol 3-kinase (PI3K) and other kinase signalling pathways. In HEK293 cells, TRPV2 proteins expressed heterologously show a species-dependent variability in thermal sensing and ligand activation. Since a 
physiological perception, depending upon the considered tissue, the TRPV2 channels has been also associated to several functions. Although, several reports on the TRPV2 channels stress towards the TRPV2 functioning instead as a mechanoreceptor and/or osmosensor. Strikingly, TRPV2 knockout mice shows normal thermal and mechanical nociception responses (Park, Vastani et al. 2011).

When analysis of sensory ganglia development (DRG and TG) occurred, resulted in no significant differences when compared knockout tissues and wild type. In the knockout mice, the analysis of hyperalgesia and acute nociception responses to mechanical and thermal stimuli were normal. Since a chemical ligand perception, TRPV2 was expressed heterologously, studies of TRPV2-null cultured neurons showed no difference in calcium responses dependent of cannabinoid, probenecid or 2-APB when compared to wild type, although the wild type responses were highly unpredictable (Park, Vastani et al. 2011). Therefore, the TRPV2 knockout mouse analysis indicates that under physiological conditions, TRPV2 is not sensitive to these compounds. The main characteristic features of the knockout mice are reduced adult body weight, perinatal viability, and embryo. Even though the studies of the knockout mice have therefore far shed little radiance onto the physiological roles of TRPV2. Further studies derived from culturing knockout mice cells shows that macrophage function is clearly affected (Link, Park et al. 2010). This may correlate with perinatal lethality relating TRPV2 to immune activity (Santoni, Farfariello et al. 2013). At last, the physiological role of TRPV2 may be one of the most troubled and contentious among TRP channels.

\section{Modulation and pharmacology of TRPV2}

TRP and TRPV channels pharmacology has been broadly and excellently reviewed (Ramsey, Delling et al. 2006; Vriens, Appendino et al. 2009; Ferrer-Montiel, Fernández-Carvajal et al. 2012). Modulation of TRPV2 channels can be regulated by the several other compounds. Still, little of them are precise, and mainly show species dependence in their efficiency on TRPV2. The fact is that the TRPV2 channels was originally projected as a noxious heat thermosensor involved in nociception. This may have mislead the TRPV2 deorphanizing attempts to find endogenous or specific modulators. Other additional physiological modulators will be described in consequent sections.

\section{Role of TRPV2 in nervous system}

TRPV2 channels are expressed in mouse DRG and spinal motor (Shibasaki, Murayama et al. 2010). According to this expression pattern and the localization of TRPV2, showed that TRPV2 involved in the development of axon outgrowth. Tominaga et al. observed that TRPV2 channels activation can also be occurred by the membrane stretching and their is subsequent increase in the intracellular $\mathrm{Ca}^{2+}$ concentration in developing neurons exerted by axon outgrowth (Shibasaki, Murayama et al. 2010). Although, TRPV2 co-localized with substance $P$ in mice (Yamamoto and Taniguchi 2005). TRPV2 activation by the cannabinoid derivatives leads to the CGRP release (Qin, Neeper et al. 2008). In the main cultures of myenteric neurons, TRPV2 activation and currents generated were invoked by probenecid, 2-APB, or lysophospholipids and stretch (Mihara, Boudaka et al. 2010). From the identical study, we also found that TRPV2 antagonists like Tranilast and nitric oxide synthase inhibitors can also inhibit the intestine relaxation. Moreover, muscle contraction is also inhibited by the TRPV2 agonists, but there is no involvement of nitric oxide synthase inhibitor, indicating that TRPV2 also play role in downstream nitric oxide activation. At last, this study therefore suggests that sympathetic muscles activity can also be controlled by TRPV2 like those of the intestinal track. In in vivo, the nitric oxide signalling pathway modulates TRPV2 function. Basbaum and Julius groups came to the conclusion is that in contrast to TRPV1, the widely distributed TRPV2 channel in the spinal cord indicates that except nociception, TRPV2 plays several 
physiological roles (Lewinter, Skinner et al. 2004).

TRPV2 has been identified as a highest expressing TRPV channel in the neurons of transcriptome of developing and mature mouse forebrain in the brain region (Cahoy, Emery et al. 2008). Within the mouse brain, this in contrast to TRPV1 study with GFP-reporter transgenic lines initiate that TRPV2 expression is restricted to the hypothalamus (Cavanaugh, Chesler et al. 2011). TRPV2 expression and distribution in rat fore- and hind brain has been recently published (Nedungadi, Dutta et al. 2012). Within this study, they observed the TRPV2 expression in a number of brain structures important to autonomic functions and osmoregulation. From this detailed work we can conclude that TRPV2 plays central role in different osmosensory mechanisms, together with osmotic balance, autonomous regulation, somatosensation, food and fluid intake, and cardiovascular functions (Muraki, Iwata et al. 2003; Nedungadi, Carreño et al. 2012; Nedungadi, Dutta et al. 2012).

\section{Role of TRPV2 in endocrine system}

In the endocrine system, TRPV2 expression is also implicated in it. It is most specifically implicated in the pancreas and insulin secretion. As earlier we have studied that TRPV2 is a calcium permeable channel. Translocate this calcium permeable channel from intracellular surface to the plasma membrane in response to IGF-1 was cloned by Kojima lab (Kanzaki, Zhang et al. 1999). By making the use of various inhibitors, knockouts and knockdowns, they determined that translocation of TRPV2 leads to the downstream of insulin signalling pathways and also lead to the upstream of insulin secretion and cell growth. This study suggests that for insulin secretion, TRPV2 is part of an autocrine positive feedback loop. In addition, TRPV2dependent intracellular increase in $\mathrm{Ca}^{2+}$ concentration leads to the glucose-induced insulin exocytosis (Aoyagi, Ohara-Imaizumi et al. 2010). It is fascinating to hypothesize that translocation of TRPV2 to the plasma membrane could be correspondingly mediate throughthe alteration of TRPV2 glycosylation.

\section{Role of TRPV2 in immunity}

Spleen, the immune-related tissues, TRPV2 is also expressed in it (Caterina, Rosen et al. 1999). TRPV2 expression have also been detected in number of macrophage populations, such as in lung alveolar macrophages, liver Kupffer cells, and skin epidermal Langerhans cells (Link, Park et al. 2010). In lymphocytes, TRPV2 expression has been identified (Saunders, Kunde et al. 2007). Turner Lab studies show that TRPV2 activation by physical stimuli leading to mast-cell degranulation and leads to the calcium entry (Stokes, Shimoda et al. 2004; Stokes, Wakano et al. 2005). They confirmed that ACBD3 (Acyl CoA binding domain protein) and A kinase adapter protein (AKAP)-like protein having a direct interaction with the TRPV2 channels. Protein kinase can also leads to the phosphorylation of the TRPV2 channels. In mast cells, TRPV2 activation can also be mediated through the protein kinase $A$ signalling pathway. In a human cell line (HMC1), mast cell degranulation mediated by the TRPV2 in response to several physical and chemical stimuli, such as temperature $\left(>50^{\circ} \mathrm{C}\right)$, red light, and mechanical stress (Zhang, Spielmann et al. 2012). In a human cell line, thermal responses mediated by TRPV2 contradicts earlier explanation that noxious heat temperatures having no effect on the human TRPV2 channels (Neeper, Liu et al. 2007).

\section{Role of TRPV2 in cancer}

In the several cancer types and cell lines, we have founded the overexpression of TRPV2 at the protein levels and mRNA (Lehen'kyi and Prevarskaya 2012). TRPV2 expression in cancer remains unspoken. In the liver cancers, higher expression of TRPV2 has been expressed in the human hepatic carcinoma cells (HepG2) (Vriens, Janssens et al. 2004). A higher level of TRPV2 channels has been founded in the cirrhotic liver as well as in the well-differentiated hepatic tumours, when compared with the poorly differentiated tumours. This results signifying that the 
TRPV2 having a potential role as a prognostic marker (Liu, Xie et al. 2010). TRPV2 also having role into the bladder cancers. mRNA and protein levels of TRPV2 were detected in normal tissue as well as in the urothelial carcinoma patients (Caprodossi, Lucciarini et al. 2008). Higher levels of TRPV2 mRNA was founded in a poorly differentiated bladder cell line when compared with the welldifferentiated line (Yamada, Ueda et al. 2010). In addition, the carcinoma cells which are having higher levels of TRPV2 undergo apoptosis when comes in contact with the TRPV2 agonist. In prostate cancer, high levels of TRPV2 expression were found in the metastatic cancers when comparison occurred with the solid tumours. From this we can conclude that for prostate cancer, TRPV2 can work as a potential marker (Monet, Lehen'kyi et al. 2010). Translocation of TRPV2 stimulating prostate cancer cell migration mediated through the Lysophospholipids to the plasma membrane, but not cell growth (Monet, Gkika et al. 2009). In the cancer cell migration, TRPV2 plays a role same as it play in the macrophages.

\section{TRPV3}

TRPV3 mainly shares about $40 \%$ identity with TRPV1. According to sequence homology, TRPV3 was cloned (Nilius, Vriens et al. 2004). TRPV3 act as a thermosensor for the non-noxious heat. It can responds to the natural compounds which are obtained from the plants such as thyme and camphor (Xu, Delling et al. 2006).

\section{Permeation}

In HEK293 cells, TRPV3 is overexpressed. It constitutes of heat activated currents and these are cation-selective having a $\mathrm{P}_{\mathrm{Ca}} / \mathrm{P}_{\mathrm{Na}}$ value of 2.6. Single-channel conductance of TRPV3 in divalent-free conditions is about $190 \mathrm{pS}(\mathrm{Xu}$, Ramsey et al. 2002).

\section{Activation}

TRPV3 is also activated by several natural compounds and warm temperatures. In response to repetitive applications of heat, TRPV3 gets sensitize (Peier, Reeve et al.
2002). Activity of TRPV3 is also sensitive to the temperature change and leads to reduction in accelerated activation kinetics and thermal threshold following an initial heat stimulus (Xu, Ramsey et al. 2002).

\section{Physiological Role}

TRPV3 play important role in thermosensation. TRPV3(-/-)mice responses to warm and noxious heat. The other sensory modalities are not dramatically diminished (Moqrich, Hwang et al. 2005). In the two mutant rodent strains, which are hairless. Under the normal conditions, the WBN/Kob-Ht rats and the autosomal dominant DS-Nh (no-hair) mouse were developed in atopic dermatitis (AD) (Asakawa, Yoshioka et al. 2006). Hence, TRPV3 is involved in the hairlessness in relation to the dermatitis; enhance the quantity of mast cells and hyperkeratosis in skin lesions. Increase in the levels ofhistamine is the main cause behind the hairlessness (Hossen, Fujii et al. 2005).

\section{TRPV 4}

TRPV4 is a channel with a pervasive multiplicity of diverse activation mechanisms. TRPV4, member of the TRPV subfamily expressed broadly in neuronal and nonneuronal cells. TRPV4 is a active $\mathrm{Ca}^{2+}$ permeable channel and can modulated by the non-noxious heat (Lee, Iida et al. 2005), and can also be modulated by the pressure and hypo tonicity(Mizuno, Matsumoto et al. 2003; Suzuki, Mizuno et al. 2003). TRPV4 is also associated with the hearing-loss (Tabuchi, Suzuki et al. 2005). About 40.9\% sequence identity was identified between the TRPV4 and TRPV1. It was the channel firstly identified as an osmosensor(Liedtke, Choe et al. 2000). TRPV4 activation can be occurred through the various physical and chemical stimuli (Nilius, Vriens et al. 2004), including high temperature (Güler, Lee et al. 2002), any mechanical stimulation (Gao, $\mathrm{Wu}$ et al. 2003), endocannabinoids(Watanabe, Vriens et al. 2003) and bisandrographolide A (BAA) (Smith, Maloney et al. 2006). 
While from its discovery, in various cells/tissues, many functions of TRPV4 has been explored. Based on the genetic TRPV4 deficiency model, the best categorized functions of TRPV4 are regulation in CNS (Liedtke and Friedman 2003), osmolarity sensing, bone remodelling (Masuyama, Vriens et al. 2008) and thermosensation(Lee, Iida et al. 2005; LEE, IIDA et al. 2005). Beside this, in the cellular models, the potential role of TRPV4 have been suggested in epithelial cells, endothelial cells, bladder urothelium and smooth muscle cells where as for these required more in vivo studies for the physiological importance (Everaerts, Nilius et al. 2010).

\section{Molecular Structure}

TRPV4 consists of 871 amino acid residues. On chromosome 12q23-q24.1, TRPV4 gene is located and having 15 exons. TRPV4 channel mainly having 4 ankyrin repeat domains and it may leads to the formation of homotetramers. The localization of the proline-rich-domain under the downstream of the first ankyrin repeat (Cuajungco, Grimm et al. 2006) and this may show involvement in TRPV4 mechanosensing (D'hoedt, Owsianik et al. 2008).

TRPV4 having basically five splice variants. Mainly widely studies form is TRPV4a which is having a full length whereas TRPV4b do not having the exon $6(\Delta 384-444$ aa), TRPV4c lacking the exon 5, TRPV4d having a exon 2 deletion from inside and TRPV4e also lacks in exon 5 and 7. Asn651 is a glycosylation site which is closer to the pore region. This glycosylation site has been identified as it can be used for the recovery from the membrane which leads to increase in the expression of cell surface (Arniges, Fernández-Fernández et al. 2006; Vázquez and Valverde 2006)

\section{Permeation and Biophysics}

TRPV4 is a $\mathrm{Ca}^{2+}$ permeable non-selective cation channels, having a rectifying currentvoltage relationship. TRPV4 having moderate permeability and selectivity for $\mathrm{Ca}^{2+}$ in the range of $\mathrm{P}_{\mathrm{Ca}} / \mathrm{P}_{\mathrm{Na}}=6-10$. Rectification is lost in the absence of extracellular $\mathrm{Ca}^{2+}$. The selectivity of the TRPV4 channel is determined by the methionine residue and two aspartate residues in the pore region of the channel (Met680, Asp672 and Asp682) (Voets, Prenen et al. 2002). At positive membrane potentials, Single channel conductance was in the range of $100 \mathrm{pS}$. At the negative membrane potentials, single channel conductance was between 30 and 60pS (Watanabe, Vriens et al. 2002).

\section{Activation}

Activation of TRPV4 occurred by various physical and chemical stimuli. The physical stimuli includes cell swelling, moderate heat $\left(>24^{\circ} \mathrm{C}\right)$ and mechanical stimuli whereas the chemical stimuli includes endocannabinoids, 4 $\alpha$-PDD, arachidonic acid (Watanabe, Davis et al. 2002; Watanabe, Vriens et al. 2002; Nilius, Watanabe et al. 2003; Nilius, Vriens et al. 2004).

Activation of TRPV4 is also occurred by increasing the temperature between $25^{\circ} \mathrm{C}$ and $34^{\circ} \mathrm{C}$. Endothelial cells and keratinocytes, both show an endogenous current with parallel threshold (Watanabe, Vriens et al. 2002; Chung, Lee et al. 2003; Chung, Lee et al. 2004). TRPV4 activation at $\sim 27^{\circ} \mathrm{C}$ by heat shows that TRPV4 is normally activated at body temperature.

TRPV4 activation is caused by PLA2 activation through cell swelling. From membrane lipids, release of arachidonic acid is mediated through PLA2 and cytochrome P450 (CYP) leads to the metabolism of arachidonic acid. The formation of epoxyeicosatrienoic acids (EETs) directly leads to the activation of TRPV4. Within pore region, Asn651 and Gln mutation leads to increase the TRPV4 membrane insertion and potentiates activation of TRPV4 through cell swelling (Watanabe, Vriens et al. 2003; Vriens, Owsianik et al. 2005; Xu, Fu et al. 2006).

\section{Conclusion}

Ion channels of TRP family having important role in the sensory mechanism of animals. Indeed, it has been found that TRPV subfamily, namely TRPV1 to TRPV4, reacts to thermal, mechanical, or painful stimuli. As nociceptors, TRPV1 to TRPV can be used as an important target for pain treatment. TRPV1 in sensory 
nociceptors, is well established novel target for the treatment of pain. TRPV1 antagonism represents several mechanisms to get relief from pain. In the preclinical study, the data indicates that TRPV1 antagonist provides a useful therapeutic option for urinary incontinence, pancreatitis, cough, and migraine.

In this review, we clearly illustrate that TRP channels superfamily are sensitive to modulation by exogenous small molecules. However, endogenous ligands make them still substantially "orphan" receptors. Moreover, the pharmacology of these channels except TRPV1 is scrubby, and selective molecular tools (ligands, blockers, and sensitizers) are missing but urgently required. In addition, information on the regions and amino acids involved in TRPV function is still on research. TRPV subfamily members play an important role in peripheral nociception. Ligand binding region could be used to rationally design novel antinociceptive or anti-inflammatory agents. These could shed radiance on the structural determinants of TRPV functionality. Finally, the physiology and regulation of vanilloid receptors increases in our knowledge. This special issue is also intended to describe these channels as novel and important targets for the development of new drugs.

Abbreviations: Transient receptor potential vanilloid (TRPV), Osmotic avoidance abnormal family member 9 (OSM 9), dorsal root ganglion (DRG), transient receptor potential (TRP), ankyrin repeat domain (ARD), protein kinase $\mathrm{C}$ (PKC), nerve growth factor (NGF), neurotropin 3 (NT3), insulin growth factor 1 (IGF-1), serum- and glucocorticoid dependent kinase 1 (SGK1), trigeminal ganglion (TG), nodose ganglion (NG), periaqueductal gray (PAG), dorsal motor nucleus of the vagal complex (DMV), resiniferatoxin (RTX), rat gastric wall (RGM1), gastroesophageal reflux disease (GERD), recombinase gene activator (RGA), vanilloidreceptor-like protein 1 (VRL-1), growth-factor regulated channel (GRC), Osm-9-like TRP channel 2 (OTRPC2), transmembrane domain (TMD), N-glycosylation consensus motif (NXT/S), Golgi-localized spectrin-repeat containing protein 56 (GSRP-56), synaptic nuclear envelope protein-1 (Syne-1), TRP channel interacting protein (TRIP), phosphatidylinositol 3-kinase (PI3K), 2aminoethoxydiphenyl borate (2-APB), Dyphenylboronic anhydride (DPBA), Acyl CoA binding domain protein (ACBD3), A kinase adapter protein (AKAP), human cell line (HMC-1), human hepato carcinoma cells (HepG2), atopic dermatitis (AD), bisandrographolide A epoxyeicosatrienoic acids (EETs)

Table 1:

\begin{tabular}{|l|l|}
\hline TRP channels & Human members \\
\hline TRPA & Having 1 human member \\
\hline TRPC & Having 6 human members, plus 1 human pseudogene \\
\hline TRPM & Having 8 human members \\
\hline TRPML & Having 3 human members \\
\hline TRPN & Having no human member \\
\hline TRPP & Having 3 human members \\
\hline TRPV & Having 6 human members \\
\hline
\end{tabular}


Table 2:

\begin{tabular}{|c|c|c|}
\hline TRPV family members & Permeation & Proposed activation mechanisms \\
\hline TRPV1 & nonselective, $\mathrm{Ca}^{2+}$ permeable & $\begin{array}{l}\text { DAG, OAG, } 20 \text {-HETE, } \\
\text { Depolarization, heat }\left(\geq 43^{\circ} \mathrm{C}\right) \text {, low } \mathrm{pH} \\
\text { ( } \leq 5.9) \text {, vanilloids, } \\
\text { endovanilloids, protein kinase C } \\
\text { (PKC), anandamide, } \\
\text { nerve growth factor (NGF), } \\
\text { neurotropin } 3 \text { (NT3), } \\
\text { sensitization by ethanol, } \\
\text { spermine. }\end{array}$ \\
\hline TRPV2 & $\begin{array}{l}\text { nonselective } \mathrm{Ca}^{2+} \\
\text { permeable }\end{array}$ & $\begin{array}{l}\text { Noxious heat }\left(>53^{\circ} \mathrm{C}\right), \text { mechanical } \\
\text { (stretch, swelling),PKC, } \mathrm{PI}(3) \mathrm{K}, \\
\text { growth factors, insulin growth factor } 1 \\
(\mathrm{IGF}-1), 2-\mathrm{APB}\end{array}$ \\
\hline TRPV3 & $\begin{array}{l}\text { nonselective } \mathrm{Ca}^{2+} \\
\text { permeable }\end{array}$ & $\begin{array}{l}\text { Heat }\left(23-29^{\circ} \mathrm{C}\right), \mathrm{PKC} \text {, camphor, } \\
\text { carvacrol, } 2-\mathrm{APB} \text {, voltage dependent }\end{array}$ \\
\hline TRPV4 & $\begin{array}{l}\text { nonselective } \mathrm{Ca}^{2+} \\
\text { permeable }\end{array}$ & $\begin{array}{l}\text { Moderate heat }\left(>24^{\circ} \mathrm{C}\right) \text {, cell swelling, } \\
\text { shear stress, PLA2 activation, PKC, } \\
\text { PAR2, anandamide. }\end{array}$ \\
\hline TRPV5 & $\begin{array}{l}\text { highly } \mathrm{Ca}^{2+} \\
\text { selective }\end{array}$ & $\begin{array}{l}\text { Hyperpolarization, voltage dependent } \\
\text { block by } \mathrm{Mg}^{2+} \text {, serum- and } \\
\text { glucocorticoid dependent kinase } 1 \\
\text { (SGK1), serine/threonine kinase } \\
\text { WNK4 }\end{array}$ \\
\hline TRPV6 & $\begin{array}{l}\text { highly } \mathrm{Ca}^{2+} \\
\text { selective }\end{array}$ & $\begin{array}{l}\text { Hyperpolarization, voltage dependent } \\
\text { block by } \mathrm{Mg}^{2+}, \mathrm{Src}\end{array}$ \\
\hline
\end{tabular}

\begin{tabular}{|ll|l|l|ll|}
\hline \multicolumn{7}{c|}{ TRP Channel Super family } & & \\
\hline \multicolumn{7}{|c|}{} \\
\hline
\end{tabular}




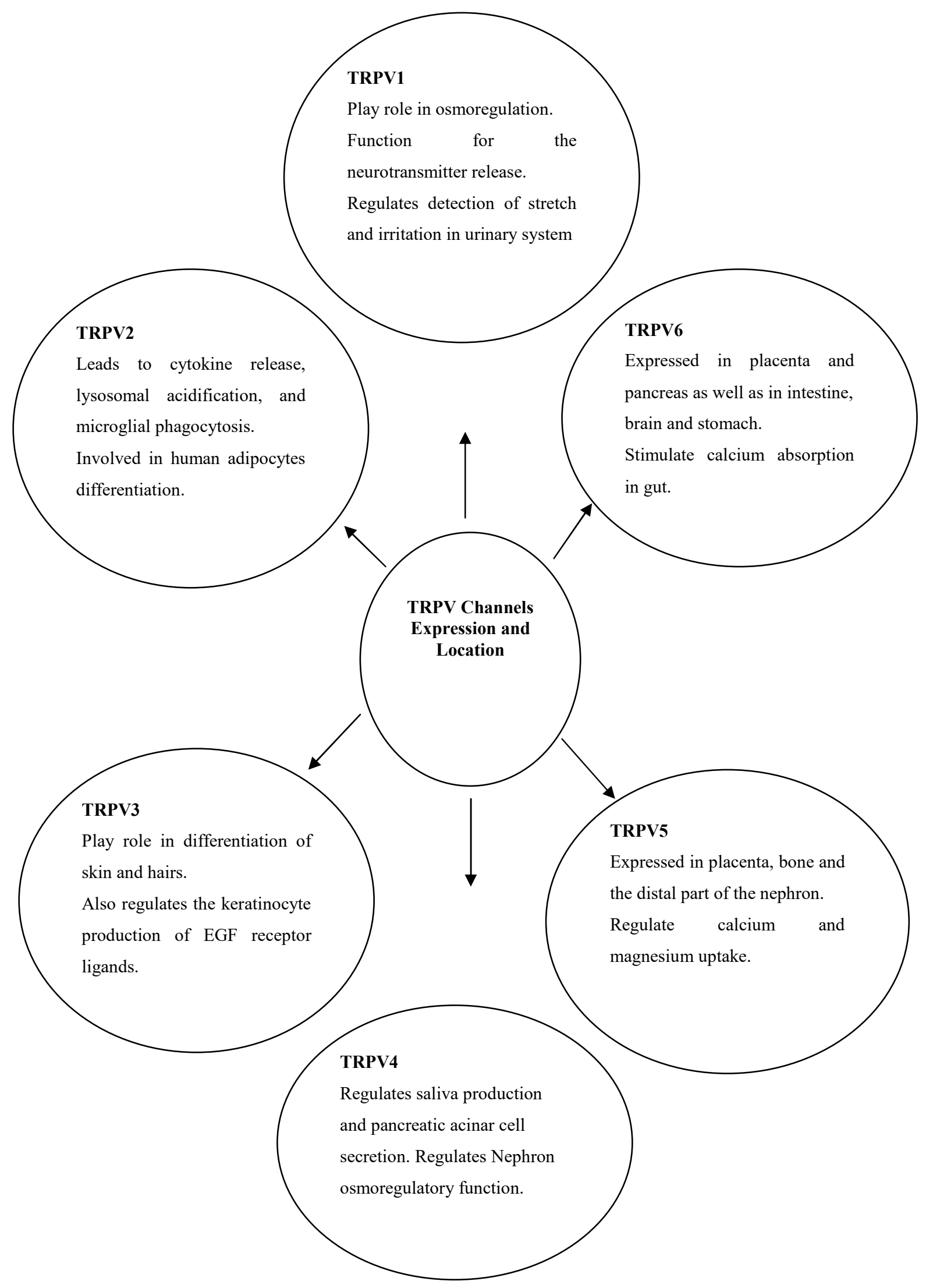




\section{References}

1. Senning, E. and S.E. Gordon, Spatiotemporal Behavior of TRPV1 Channels in the Plasma Membrane. Biophysical Journal. 2011; 100(3): 108a.

2. Liedtke, W., 22 TRPV Channels' Function in Osmo-and Mechanotransduction. TRP Ion Channel Function in Sensory Transduction and Cellular Signaling Cascades. 2006;2006: 303.

3. Ramsey, I.S., M. Delling, and D.E. Clapham, An introduction to TRP channels. Annu. Rev. Physiol., 2006. 68: p. 619-647.

4. Montell, C. and G.M. Rubin, Molecular characterization of the Drosophila trp locus: a putative integral membrane protein required for phototransduction. Neuron, 1989. 2(4): p. 1313-1323.

5. Almén, M.S., et al., Mapping the human membrane proteome: a majority of the human membrane proteins can be classified according to function and evolutionary origin. BMC biology, 2009. 7(1): p. 50.

6. Venkatachalam, K. and C. Montell, TRP channels. Annual review of biochemistry, 2007. 76: p. 387.

7. Inada, H. and R. Gaudet, Structural comparison of Ankyrin repeat domain of TRPV channels. Biophysical Journal, 2011. 100(3): p. 108a.

8. Montell, C., The TRP superfamily of cation channels. Science Signaling, 2005. 2005(272): p. re3-re3.

9. Clapham, D.E., Mammalian TRP channels. Cell, 2007. 129(1): p. 220.

10. Hellwig, N., et al., Homo-and heteromeric assembly of TRPV channel subunits. J Cell Sci, 2005. 118(5): p. 917-928.

11. Owsianik, G., et al., Permeation and selectivity of TRP channels. Annu. Rev. Physiol., 2006. 68: p. 685-717.

12. Verkhratsky, A., R.C. Reyes, and V. Parpura, TRP channels coordinate ion signalling in astroglia, in Reviews of Physiology, Biochemistry and Pharmacology 166. 2013, Springer. p. 1-22.

13. Inoue, R., H. Morita, and Y. Ito, Newly emerging $\mathrm{Ca} 2+$ entry channel molecules that regulate the vascular tone. Expert opinion on therapeutic targets, 2004. 8(4): p. 321-334.

14. Birder, L. and K.-E. Andersson, Urothelial signaling. Physiological reviews, 2013. 93(2): p. 653-680.

15. Everaerts, W., et al., On the origin of bladder sensing: $\operatorname{Tr}$ (i) ps in urology. Neurourology and urodynamics, 2008. 27(4): p. 264-273.

16. Holzer, P., TRP channels in the digestive system. Current pharmaceutical biotechnology, 2011. 12(1): p. 24.

17. Santoni, G., et al., The role of transient receptor potential vanilloid type-2 ion channels in innate and adaptive immune responses. Front Immunol, 2013. 4: p. 34.

18. Moorea, C., et al., UVB radiation generates sunburn pain and affects skin by activating epidermal TRPV4 ion channels and triggering endothelin-1 signaling. PNAS, 2013. 110(38).

19. Wilson, S.R., et al., The epithelial cellderived atopic dermatitis cytokine TSLP activates neurons to induce itch. Cell, 2013. 155(2): p. 285-295.

20. Bautista, D.M., M. Pellegrino, and M. Tsunozaki, TRPA1: A gatekeeper for Inflammation. Annual review of physiology, 2013. 75: p. 181.

21. Geppetti, P., et al., The concept of neurogenic inflammation. BJU international, 2008. 101(s3): p. 2-6.

22. Cheng, X., et al., TRP channel regulates EGFR signaling in hair morphogenesis and skin barrier formation. Cell, 2010. 141(2): p. 331-343.

23. Che, H., et al., Functional TRPV and TRPM channels in human preadipocytes. Pflügers Archiv- 
European Journal of Physiology, 2014. 466(5): p. 947-959.

24. Gharat, L. and A. Szallasi, Medicinal chemistry of the vanilloid (Capsaicin) TRPV1 receptor: Current knowledge and future perspectives. Drug Development Research, 2007. 68(8): p. 477-497.

25. Knotkova, H., M. Pappagallo, and A. Szallasi, Capsaicin (TRPV1 Agonist) therapy for pain relief: farewell or revival? The Clinical journal of pain, 2008. 24(2): p. 142.

26. Wong, G.Y. and N.R. Gavva, Therapeutic potential of vanilloid receptor TRPV1 agonists and antagonists as analgesics: recent advances and setbacks. Brain research reviews, 2009. 60(1): p. 267-277.

27. Ferrer-Montiel, A., et al., Advances in modulating thermosensory TRP channels. Expert opinion on therapeutic patents, 2012. 22(9): p. 999-1017.

28. Matsumoto, K., et al., Localization of TRPV1 and contractile effect of capsaicin in mouse large intestine: high abundance and sensitivity in rectum and distal colon. American journal of physiology. Gastrointestinal and liver physiology, 2009. 297(2): p. G348-60.

29. Geppetti, P. and M. Trevisani, Activation and sensitisation of the vanilloid receptor: role in gastrointestinal inflammation and function. British journal of pharmacology, 2004. 141(8): p. 13131320.

30. Matthews, P.J., et al., Increased capsaicin receptor TRPV1 nerve fibres in the inflamed human oesophagus. European journal of gastroenterology \& hepatology, 2004. 16(9): p. 897-902.

31. Bhat, Y.M. and K. Bielefeldt, Capsaicin receptor (TRPV1) and non-erosive reflux disease. European journal of gastroenterology \& hepatology, 2006. 18(3): p. 263-270.
32. Fujino, K., et al., Attenuation of acid induced oesophagitis in VR-1 deficient mice. Gut, 2006. 55(1): p. 34-40.

33. Spencer, N.J., et al., Identification of capsaicin-sensitive rectal mechanoreceptors activated by rectal distension in mice. Neuroscience, 2008. 153(2): p. 518-534.

34. Jones, R.C.W., L. Xu, and G. Gebhart, The mechanosensitivity of mouse colon afferent fibers and their sensitization by inflammatory mediators require transient receptor potential vanilloid 1 and acid-sensing ion channel 3. The Journal of neuroscience, 2005. 25(47): p. 10981-10989.

35. Wang, Z.-Y., et al., Lack of TRPV1 inhibits cystitis-induced increased mechanical sensitivity in mice. Pain, 2008. 139(1): p. 158-167.

36. Christoph, T., et al., Silencing of vanilloid receptor TRPV1 by RNAi reduces neuropathic and visceral pain in vivo. Biochemical and biophysical research communications, 2006. 350(1): p. 238-243.

37. Sugiura, T., K. Bielefeldt, and G.F. Gebhart, Mouse colon sensory neurons detect extracellular acidosis via TRPV1. American Journal of Physiology-Cell Physiology, 2007. 292(5): p. C1768C1774.

38. Yiangou, Y., et al., Vanilloid receptor 1 immunoreactivity in inflamed human bowel. The Lancet, 2001. 357(9265): p. 1338-1339.

39. Winston, J., et al., The vanilloid receptor initiates and maintains colonic hypersensitivity induced by neonatal colon irritation in rats. Gastroenterology, 2007. 132(2): p. 615627.

40. Keszthelyi, D., et al., Alterations in mucosal neuropeptides in patients with irritable bowel syndrome and ulcerative colitis in remission: a role in pain symptom generation? European Journal of Pain, 2013. 17(9): p. 1299-1306. 
41. Avelino, A., et al., Transient receptor potential channels in bladder function. Acta Physiologica, 2013. 207(1): p. 110-122.

42. Lazzeri, M., P. Beneforti, and D. Turini, Urodynamic effects of intravesical resiniferatoxin in humans: preliminary results in stable and unstable detrusor. The Journal of urology, 1997. 158(6): p. 2093-2096.

43. Silva, C., et al., Bladder sensory desensitization decreases urinary urgency. BMC urology, 2007. 7(1): p. 9.

44. Watanabe, T., et al., Intravesical resiniferatoxin for patients with neurogenic detrusor overactivity. International journal of urology, 2004. 11(4): p. 200-205.

45. Silva, C., et al., A light-and electron-microscopic histopathological study of human bladder mucosa after intravesical resiniferatoxin application. BJU international, 2001. 88(4): p. 355360.

46. Silva, C., M.-E. Rio, and F. Cruz, Desensitization of Bladder Sensory Fibers by Intravesical Resiniferatoxin, a Capsaicin Analog: Long-Term Results for the Treatment of Detrusor Hyperreflexia. European urology, 2000. 38(4): p. 444-452.

47. Silva, C., et al., Urodynamic effect of intravesical resiniferatoxin in patients with neurogenic detrusor overactivity of spinal origin: results of a double-blind randomized placebo-controlled trial. European urology, 2005. 48(4): p. 650655.

48. Barnhill, J.C., et al., RGA protein associates with a TRPV ion channel during biosynthesis and trafficking. Journal of cellular biochemistry, 2004. 91(4): p. 808-820.

49. Park, U., et al., TRP vanilloid 2 knockout mice are susceptible to perinatal lethality but display normal thermal and mechanical nociception. The Journal of
Neuroscience, 2011. 31(32): p. 1142511436.

50. Caterina, M.J., et al., A capsaicinreceptor homologue with a high threshold for noxious heat. Nature, 1999. 398(6726): p. 436-441.

51. Kanzaki, M., et al., Translocation of a calcium-permeable cation channel induced by insulin-like growth factor-I. Nature cell biology, 1999. 1(3): p. 165170.

52. Harteneck, C., T.D. Plant, and G. Schultz, From worm to man: three subfamilies of TRP channels. Trends in neurosciences, 2000. 23(4): p. 159-166.

53. Cheng, W., et al., Thermosensitive TRPV channel subunits coassemble into heteromeric channels with intermediate conductance and gating properties. The Journal of general physiology, 2007. 129(3): p. 191-207.

54. Liapi, A. and J.N. Wood, Extensive co-localization and heteromultimer formation of the vanilloid receptor-like protein TRPV2 and the capsaicin receptor TRPV1 in the adult rat cerebral cortex. European Journal of Neuroscience, 2005. 22(4): p. 825-834.

55. Saito, S. and R. Shingai, Evolution of thermoTRP ion channel homologs in vertebrates. Physiological genomics, 2006. 27(3): p. 219-230.

56. McCleverty, C.J., et al., Crystal structure of the human TRPV2 channel ankyrin repeat domain. Protein Science, 2006. 15(9): p. 2201-2206.

57. Neeper, M.P., et al., Activation Properties of Heterologously Expressed Mammalian TRPV2 EVIDENCE FOR SPECIES DEPENDENCE. Journal of Biological Chemistry, 2007. 282(21): p. 15894-15902.

58. Jin, X., J. Touhey, and R. Gaudet, Structure of the N-terminal ankyrin repeat domain of the TRPV2 ion channel. Journal of Biological Chemistry, 2006. 281(35): p. 2500625010. 
59. Michaely, P., et al., Crystal structure of a 12 ANK repeat stack from human ankyrinR. The EMBO journal, 2002. 21(23): p. 6387-6396.

60. Krogh, A., et al., Predicting transmembrane protein topology with a hidden Markov model: application to complete genomes. Journal of molecular biology, 2001. 305(3): p. 567-580.

61. Boukalova, S., et al., Conserved residues within the putative $\mathrm{S} 4-\mathrm{S} 5$ region serve distinct functions among thermosensitive vanilloid transient receptor potential (TRPV) channels. Journal of Biological Chemistry, 2010. 285(53): p. 41455-41462.

62. Jahnel, R., et al., Dual expression of mouse and rat VRL-1 in the dorsal root ganglion derived cell line F-11 and biochemical analysis of VRL-1 after heterologous expression. European Journal of Biochemistry, 2003. 270(21): p. 4264-4271.

63. Morenilla-Palao, C., et al., Lipid raft segregation modulates TRPM8 channel activity. Journal of Biological Chemistry, 2009. 284(14): p. 92159224.

64. Erler, I., et al., Trafficking and assembly of the cold-sensitive TRPM8 channel. Journal of Biological Chemistry, 2006. 281(50): p. 3839638404.

65. Lin, Y. and Z. Sun, Antiaging gene Klotho enhances glucose-induced insulin secretion by up-regulating plasma membrane levels of TRPV2 in MIN6 $\beta$-cells. Endocrinology, 2012. 153(7): p. 3029-3039.

66. Penna, A., et al., PI3-kinase promotes TRPV2 activity independently of channel translocation to the plasma membrane. Cell calcium, 2006. 39(6): p. 495-507.

67. Mercado, J., et al., Ca2+-dependent desensitization of TRPV2 channels is mediated by hydrolysis of phosphatidylinositol 4, 5-bisphosphate.
The Journal of Neuroscience, 2010. 30(40): p. 13338-13347.

68. Holakovska, B., et al., Characterization of calmodulin binding domains in TRPV2 and TRPV5 C-tails. Amino acids, 2011. 40(2): p. 741-748.

69. García-Sanz, N., et al., Identification of a tetramerization domain in the $\mathrm{C}$ terminus of the vanilloid receptor. The Journal of Neuroscience, 2004. 24(23): p. 5307-5314.

70. Stokes, A.J., et al., Formation of a physiological complex between TRPV2 and RGA protein promotes cell surface expression of TRPV2. Journal of cellular biochemistry, 2005. 94(4): p. 669-683.

71. Phelps, C.B., et al., Differential regulation of TRPV1, TRPV3, and TRPV4 sensitivity through a conserved binding site on the ankyrin repeat domain. Journal of Biological Chemistry, 2010. 285(1): p. 731-740.

72. Myers, B.R., C.J. Bohlen, and D. Julius, A yeast genetic screen reveals a critical role for the pore helix domain in TRP channel gating. Neuron, 2008. 58(3): p. 362-373.

73. Gordon-Shaag, A., W.N. Zagotta, and S.E. Gordon, Mechanism of $\mathrm{Ca} 2+-$ dependent desensitization in TRP channels. Channels, 2008. 2(2): p. 125129.

74. Shin, Y.-C., et al., TRIP Database: a manually curated database of proteinprotein interactions for mammalian TRP channels. Nucleic acids research, 2010: p. gkq814.

75. Shin, Y.-C., et al., TRIP database 2.0: a manually curated information hub for accessing TRP channel interaction network. PloS one, 2012. 7(10): p. e47165.

76. Link, T.M., et al., TRPV2 has a pivotal role in macrophage particle binding and phagocytosis. Nature immunology, 2010. 11(3): p. 232-239.

77. Vriens, J., G. Appendino, and B. Nilius, Pharmacology of vanilloid transient 
receptor potential cation channels. Molecular pharmacology, 2009. 75(6): p. 1262-1279.

78. Shibasaki, K., et al., TRPV2 enhances axon outgrowth through its activation by membrane stretch in developing sensory and motor neurons. The Journal of Neuroscience, 2010. 30(13): p. 46014612.

79. Yamamoto, $\mathrm{Y}$. and K. Taniguchi, Immunolocalization of VR1 and VRL1 in rat larynx. Autonomic Neuroscience, 2005. 117(1): p. 62-65.

80. Mihara, H., et al., Involvement of TRPV2 activation in intestinal movement through nitric oxide production in mice. The Journal of Neuroscience, 2010. 30(49): p. 1653616544.

81. Lewinter, R.D., et al., Immunoreactive TRPV-2 (VRL-1), a capsaicin receptor homolog, in the spinal cord of the rat. Journal of Comparative Neurology, 2004. 470(4): p. 400-408.

82. Cahoy, J.D., et al., A transcriptome database for astrocytes, neurons, and oligodendrocytes: a new resource for understanding brain development and function. The Journal of Neuroscience, 2008. 28(1): p. 264-278.

83. Cavanaugh, D.J., et al., Trpv1 reporter mice reveal highly restricted brain distribution and functional expression in arteriolar smooth muscle cells. The Journal of Neuroscience, 2011. 31(13): p. 5067-5077.

84. Nedungadi, T.P., et al., Expression and distribution of TRPV2 in rat brain. Experimental neurology, 2012. 237(1): p. 223-237.

85. Nedungadi, T.P., et al., Region-Specific Changes in Transient Receptor Potential Vanilloid Channel Expression in the Vasopressin Magnocellular System in Hepatic Cirrhosis-Induced Hyponatraemia. Journal of neuroendocrinology, 2012. 24(4): $p$. 642-652.
86. Muraki, K., et al., TRPV2 is a component of osmotically sensitive cation channels in murine aortic myocytes. Circulation research, 2003. 93(9): p. 829-838.

87. Aoyagi, K., et al., Insulin/phosphoinositide 3-kinase pathway accelerates the glucoseinduced first-phase insulin secretion through TrpV2 recruitment in pancreatic $\beta$-cells. Biochemical Journal, 2010. 432(2): p. 375-386.

88. Saunders, C.I., et al., Expression of transient receptor potential vanilloid 1 (TRPV1) and 2 (TRPV2) in human peripheral blood. Molecular immunology, 2007. 44(6): p. 14291435.

89. Stokes, A.J., et al., A TRPV2-PKA signaling module for transduction of physical stimuli in mast cells. The Journal of experimental medicine, 2004. 200(2): p. 137-147.

90. Zhang, D., et al., Mast-cell degranulation induced by physical stimuli involves the activation of transient-receptor-potential channel TRPV2. Physiol Res, 2012. 61(1): p. 113-124.

91. Lehen'kyi, V.y. and N. Prevarskaya, Gene

Section.http://AtlasGeneticsOncology. org, 2012: p. 563.

92. Vriens, J., et al., TRPV channels and modulation by hepatocyte growth factor/scatter factor in human hepatoblastoma (HepG2) cells. Cell calcium, 2004. 36(1): p. 19-28.

93. Liu, G., et al., Clinical significance of transient receptor potential vanilloid 2 expression in human hepatocellular carcinoma. Cancer genetics and cytogenetics, 2010. 197(1): p. 54-59.

94. Caprodossi, S., et al., Transient receptor potential vanilloid type 2 (TRPV2) expression in normal urothelium and in urothelial carcinoma of human bladder: correlation with the pathologic stage. 
European urology, 2008. 54(3): p. 612620.

95. Yamada, T., et al., TRPV2 activation induces apoptotic cell death in human T24 bladder cancer cells: a potential therapeutic target for bladder cancer. Urology, 2010. 76(2): p. 509. e1-509. e7.

96. Monet, M., et al., Role of cationic channel TRPV2 in promoting prostate cancer migration and progression to androgen resistance. Cancer research, 2010. 70(3): p. 1225-1235.

97. Monet, M., et al., Lysophospholipids stimulate prostate cancer cell migration via TRPV2 channel activation. Biochimica et Biophysica Acta (BBA)Molecular Cell Research, 2009. 1793(3): p. 528-539.

98. Nilius, B., et al., TRPV4 calcium entry channel: a paradigm for gating diversity. American Journal of Physiology-Cell Physiology, 2004. 286(2): p. C195-C205.

99. Hossen, M.A., et al., Effect of loratadine on mouse models of atopic dermatitis associated pruritus. International immunopharmacology, 2005. 5(7): p. 1331-1336.

100. Lee, H., et al., Altered thermal selection behavior in mice lacking transient receptor potential vanilloid 4 . The Journal of neuroscience: the official journal of the Society for Neuroscience, 2005. 25(5): p. 1304.

101. Suzuki, M., et al., Impaired pressure sensation in mice lacking TRPV4. Journal of Biological Chemistry, 2003. 278(25): p. 22664-22668.

102. Mizuno, A., et al., Impaired osmotic sensation in mice lacking TRPV4. American Journal of Physiology-Cell Physiology, 2003. 285(1): p. C96-C101.

103. Tabuchi, K., et al., Hearing impairment in TRPV4 knockout mice. Neuroscience letters, 2005. 382(3): p. 304-308.

104. Nilius, B., et al., TRPV4 calcium entry channel: a paradigm for gating diversity. American Journal of
Physiology. Cell Physiology, 2004. 286(2): p. C195-205.

105. Güler, A.D., et al., Heat-Evoked Activation of the Ion Channel, TRPV4. The Journal of Neuroscience, 2002. 22(15): p. 6408-6414.

106. Gao, X., L. Wu, and R. O'Neil, Temperature-modulated diversity of TRPV4 channel gating: activation by physical stresses and phorbol ester derivatives through protein kinase $\mathrm{C}$ dependent and-independent pathways. The Journal of biological chemistry, 2003. 278(29): p. 27129.

107. Watanabe, H., et al., Anandamide and arachidonic acid use epoxyeicosatrienoic acids to activate TRPV4 channels. Nature, 2003. 424(6947): p. 434-438.

108. Smith, P.L., et al., Bisandrographolide from Andrographis paniculata Activates TRPV4 Channels. Journal of Biological Chemistry, 2006. 281(40): p. $29897-$ 29904.

109. Liedtke, W. and J. Friedman, Abnormal osmotic regulation in trpv4-/-mice. Proceedings of the National Academy of Sciences of the United States of America, 2003. 100(23): p. 1369813703.

110. Masuyama, R., et al., TRPV4-Mediated Calcium Influx Regulates Terminal Differentiation of Osteoclasts. Cell Metabolism, 2008. 3(8): p. 257-265.

111. LEE, H., et al., Altered thermal selection behavior in mice lacking transient receptor potential vanilloid 4 . The Journal of Neuroscience, 2005. 25(5): p. 1304-1310.

112. Cuajungco, M.P., et al., PACSINs bind to the TRPV4 cation channel PACSIN 3 modulates the subcellular localization of TRPV4. Journal of Biological Chemistry, 2006. 281(27): p. 1875318762.

113. D'hoedt, D., et al., Stimulus-specific modulation of the cation channel TRPV4 by PACSIN 3. Journal of 
Biological Chemistry, 2008. 283(10): p. 6272-6280.

114. Vázquez, E. and M.A. Valverde. A review of TRP channels splicing. in Seminars in cell \& developmental biology. 2006: Elsevier.

115. Arniges, M., et al., Human TRPV4 channel splice variants revealed a key role of ankyrin domains in multimerization and trafficking. Journal of Biological Chemistry, 2006. 281(3): p. 1580-1586.

116. Voets, T., et al., Molecular determinants of permeation through the cation channel TRPV4. Journal of Biological Chemistry, 2002. 277(37): p. 3370433710 .

117. Watanabe, H., et al., Heat-evoked activation of TRPV4 channels in a HEK293 cell expression system and in native mouse aorta endothelial cells. Journal of Biological Chemistry, 2002. 277(49): p. 47044-47051.
118. Nilius, B., H. Watanabe, and J. Vriens, The TRPV4 channel: structure-function relationship and promiscuous gating behaviour. Pflügers Archiv, 2003. 446(3): p. 298-303.

119. Watanabe, H., et al., Activation of TRPV4 channels (hVRL-2/mTRP12) by phorbol derivatives. Journal of Biological Chemistry, 2002. 277(16): p. 13569-13577.

120. Vriens, J., et al., Modulation of the Ca2 permeable cation channel TRPV4 by cytochrome $\mathrm{P} 450$ epoxygenases in vascular endothelium. Circulation research, 2005. 97(9): p. 908-915.

121. $\mathrm{Xu}, \mathrm{H}$., et al., Glycosylation of the osmoresponsive transient receptor potential channel TRPV4 on Asn-651 influences membrane trafficking. American Journal of Physiology-Renal Physiology, 2006. 290(5): p. F1103F1109. 